\title{
Total cross section measurements for positron scattering from acetone
}

\author{
Antonio Zecca ${ }^{1}$, Luca Chiari ${ }^{1}$, Emanuele Trainotti ${ }^{1}$, Anindya Sarkar ${ }^{2}$, Michael J Brunger ${ }^{3 *}$
}

\author{
* Correspondence: michael. \\ brunger@flinders.edu.au \\ ${ }^{3}$ ARC Centre for Antimatter-Matter \\ Studies, School of Chemical and \\ Physical Sciences, Flinders \\ University, GPO Box 2100, Adelaide, \\ SA 5001, Australia
}

\begin{abstract}
We report results from total cross section measurements for positron-acetone scattering. The energy range of these experiments was $0.2-23 \mathrm{eV}$, while the energy resolution of our positron beam was $\sim 260 \mathrm{meV}$. The present data clearly highlight the important role played by the strong permanent dipole moment and significant dipole polarisability of the acetone molecule on the low-energy scattering dynamics of this system. For positron energies above about $6 \mathrm{eV}$ the present data is found to be in quite good agreement with the only other total cross section results available in the literature from the Yamaguchi group, however, at lower energies the level of agreement is rather poor. To the best of our knowledge, no theoretical calculations are currently available for positron-acetone scattering.

PACS Codes: 34.80.Uv
\end{abstract}

\section{Introduction}

In our previous studies on water [1], tetrahydrofuran [2], 3-hydroxy-tetrahydrofuran [3] and di-hydropyran [4], all of which have significant permanent dipole moments $(\mu)$ and dipole polarisabilities $(\alpha)$ of important magnitude, we observed that for each species the total cross sections (TCSs) increased significantly in value with decreasing incident positron impact energies. In a joint collaboration with theorists, in that case for formic acid which also possesses a strong dipole moment and dipole polarisability, we were able to explicitly demonstrate [5] that the observed low energy behaviour of the TCSs was due to those $(\mu, \alpha)$ physico-chemical properties of the target molecule in question. Very recently [6] the San Diego group has also demonstrated the important role that the dipole moment plays in enhancing positron binding to molecules, clearly indicating the crucial role that it plays in positron scattering dynamics.

Acetone $\left(\mathrm{CH}_{3} \mathrm{COCH}_{3}\right)$, which plays an important role in chemistry as a solvent and as a precursor to polymers, is also known to possess a very strong dipole moment $(\mu=2.89$ D) [7] (compared with water where $\mu=1: 85 \mathrm{D}$ [8]). The origin of this can be seen in figure 1, which is a schematic diagram of the acetone molecule. Here two lone pairs of electrons are located on the oxygen atom in the carboxyl group, leading to acetone having a significant charge assymetry and thus strong permanent dipole moment. In addition, it is well known that acetone has a rather extended electron charge cloud, as evidenced by a molecular diameter of $6.16 \AA$ [9], a dipole polarisability of $6.4 \AA^{3}[7,10]$ and an effective annihilation parameter $\left(\mathrm{Z}_{\mathrm{eff}}\right)$ of 98,400 [8]. All these properties of the target, given our previous experience [1-5], would suggest that the total cross section for 


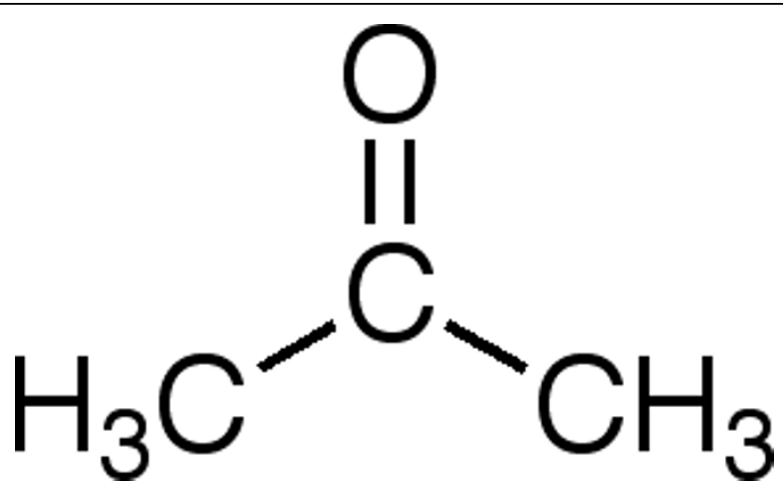

Figure 1 Acetone molecule. A schematic representation of the acetone molecule.

positron-acetone scattering might be expected to increase dramatically as you go to lower incident positron energies. However, the only available investigations into TCSs for positron-acetone scattering $[11,12]$ indicate the exact opposite. Namely they show that as you go to lower positron impact energies the TCSs decrease in magnitude. This is a puzzling result and as a consequence we revisit this system to report new measurements of total cross sections for positron scattering from acetone.

In the next section we describe our experimental apparatus and measurement techniques. Thereafter the current results are presented and discussed, before some conclusions from the present study are given.

\section{Experimental Details}

The positron spectrometer employed at the University of Trento was developed by Zecca and co-workers, and has been described in detail many times [see e.g. [1-5]]. We therefore do not repeat those details again here, except to note that a tungsten moderator of thickness $1 \mu \mathrm{m}$ or a nickel moderator of $2 \mu \mathrm{m}$ was employed in conjunction with a radioactive ${ }^{22} \mathrm{Na}$ isotope (current activity $\sim 2.2 \mathrm{mCi}$ ) and some electrostatic optics in order to produce the positron beam. Full details on the comparative performance of these moderators can be found in Zecca et al. [13], with consistent TCS results being obtained in either case. Here we note that most of the present data were collected with the tungsten moderator. We further note that it is a standard practice in our laboratory, as a check for the validity of our techniques and procedures, to perform preliminary validation measurements using targets for which the positron scattering total cross sections are considered well known. Such a standard system might be drawn from the lighter rare gases [14] or as a consequence of our recent detailed study involving molecular nitrogen (A. Zecca, L. Chiari, A. Sarkar and M. J. Brunger: Positron scattering from the isoelectronic molecules $\mathrm{N}_{2}, \mathrm{CO}$ and $\mathrm{C}_{2} \mathrm{H}_{2}$, unpublished).

The basis of all our linear transmission experiments is the Beer-Lambert law, as defined by:

$$
I_{1}=I_{0} \exp \left(\frac{-\left(P_{1}-P_{0}\right) L \sigma}{k T}\right),
$$

where $I_{1}$ is the positron beam count rate at pressure $P_{1}$, the pressure being measured with the acetone routed to the scattering cell, $k$ is Boltzmann's constant, $T$ is the 
temperature of the acetone vapour $(\mathrm{K}), \sigma$ is the total cross section, $I_{0}$ is the positron count rate at $P_{0}$, the pressure with the acetone diverted into the vacuum chamber i.e. away from the scattering cell, and $L$ is the length of the scattering region.

For a physical application of equation (1) several crucial precautions should be taken and care must be exercised during the measurements. Those considerations include minimising double-scattering events and ensuring the TCSs are independent of pressure. In addition, only a high-purity acetone sample (>99.8\%) was used (Merck). Note that while acetone is a liquid at room temperatures, it is sufficiently volatile to be able to perform our measurements. Further note that to minimise any possible impurities affecting our measurements freeze-pump-thaw cycles were employed here.

The geometrical length of the scattering region is $22.1 \pm 0.1 \mathrm{~mm}$, with apertures of $1.5 \mathrm{~mm}$ diameter at both the entrance and exit of the scattering chamber. End effects were minimised in this study by having relatively small diameter entrance and exit apertures in the scattering chamber. As a consequence we believe their contribution to the uncertainty in the value of $L$ is likely to be less than $0.2 \%$. In our application of equation (1), the value of $L$ used is always corrected to account for the path increase caused by the gyration of the positrons in the focussing axial magnetic field $(\sim 12 \mathrm{G})$ present in the scattering region. For the present study this led to the value of $L$ to be increased by $\sim 6 \%$. From a consideration of the size of the entrance and exit apertures of our scattering cell, and their separation, the angular acceptance $(\Delta \theta)$ of the Trento spectrometer is $\approx 4^{\circ}$, which compares favourably with that from the Yamaguchi spectrometer $\left(\Delta \theta \approx 7^{\circ}\right)(\mathrm{C}$. Makochekanwa, 2010, private communication) and the Detroit spectrometer $\left(\Delta \theta \approx 16^{\circ}\right)$ [15]. The gyration of the positrons can also potentially increase the angular resolution error compared to the no-field case [16]. Using some of the analytic formulae detailed in Kauppila et al. [15], but for the typical experimental conditions of our measurements, estimates of the energy-dependent angular discrimination can be obtained. We found that they varied from $\sim 17^{\circ}$ at $1 \mathrm{eV}$ positron energy to $\sim 5.4^{\circ}$ at $10 \mathrm{eV}$. This can also be corrected for, provided appropriate absolute elastic differential cross sections (theory or experimental) are available. Unfortunately, such differential cross sections are not usually available so that the TCSs we report here represent a lower bound on the exact values. We shall return to this point again in the next section.

It is very important for the energy scale to be calibrated accurately. The zero for the energy scale, in the absence of the target gas, was determined in this investigation with a retarding potential analysis of the positron beam [17]. We now believe that the error in our energy scale is of the order of $\pm 0.1 \mathrm{eV}$. Measurements repeated during the last 3-4 years show a surprising stability in the energy zero (variance $<0.05 \mathrm{eV}$ ) when using the tungsten moderator. Similar measurements performed on nickel moderators show a comparable stability. The same measurements allow us to evaluate an energy width of the positron beam of $\sim 260 \mathrm{meV}$ with an uncertainty on this determination of at most $100 \mathrm{meV}$. This value refers to the predominant case of measurements being made with the tungsten moderator. It is also crucial to accurately measure the scattering cell pressure, which we achieve with a MKS 627B capacitance manometer operated at $45{ }^{\circ} \mathrm{C}$. As the manometer temperature was different to that for the target gas in the scattering cell, thermal transpiration corrections to the pressure readings are made using the model of Takaishi and Sensui [18]. Typically this led to a maximum correction on the TCS of $3.3 \%$. One final caveat on the data we report here should be noted. 
All the experimental data are convoluted with the finite energy resolution of our positron beam $(\sim 260 \mathrm{meV})$, although the effect of this on the measured TCSs is most manifest at positron energies below $\sim 0.5 \mathrm{eV}$ which coincides with where the slope of the TCS as a function of energy is also greatest. In practice this physically implies that, when corrected for this effect, our lowest energy TCSs should be somewhat higher in magnitude than what we present here.

Finally we note that the data collection and analysis codes were driven by software developed at the University of Trento, for application on a personal computer. The positron energy range of the present TCS measurements was $0.2-23 \mathrm{eV}$, with the overall errors on our total cross sections typically being within the $5-15 \%$ range. Note that the overall errors are formed from the quadrature sum of the statistical uncertainties on our data (see table 1), the uncertainty in our thermal transpiration corrections, the uncertainty in the value of $L$ and the uncertainty in the absolute pressure readings $(\sim 0.3 \%)$, as per the manufacturer's specification. All our measurements were taken under stable positron beam conditions.

\section{Results and discussion}

In table 1 and figure 2 we present the results of our positron-acetone total cross section measurements. Note that the errors listed in table 1 and plotted in figure 2 are purely statistical and are at the one standard deviation level. Further note that the arrow in figure 2 indicates the threshold for positronium (Ps) formation. Also shown in figure 2 are the previous results from Yamaguchi University [11,12]. We advise that to the best of our knowledge no theory is currently available for this scattering system, although we are aware that relevant calculations are being considered by the Brazilian group (M. H. F. Bettega, 2010, private communication).

Table 1 Present total cross sections $\left(\times 10^{-20} \mathrm{~m}^{2}\right)$ for positron scattering from acetone

\begin{tabular}{|c|c|c|c|c|c|}
\hline $\begin{array}{c}\text { Energy } \\
(\mathrm{eV})\end{array}$ & $\begin{array}{c}\text { TCS } \\
\left(10^{-20} \mathrm{~m}^{2}\right)\end{array}$ & $\begin{array}{c}\text { TCS error } \\
\left(10^{-20} \mathrm{~m}^{2}\right) \\
( \pm 1 \sigma)\end{array}$ & $\begin{array}{c}\text { Energy } \\
(\mathrm{eV})\end{array}$ & $\begin{array}{c}\text { TCS } \\
\left(10^{-20} \mathrm{~m}^{2}\right)\end{array}$ & $\begin{array}{c}\text { TCS error } \\
\left(10^{-20} \mathrm{~m}^{2}\right) \\
( \pm 1 \sigma)\end{array}$ \\
\hline 0.20 & 149.57 & 7.21 & 6.00 & 27.42 & 3.90 \\
\hline 0.30 & 133.84 & 6.46 & 7.00 & 25.69 & 2.20 \\
\hline 0.40 & 121.13 & 6.13 & 8.00 & 25.40 & 4.57 \\
\hline 0.50 & 107.62 & 7.14 & 9.00 & 24.95 & 4.30 \\
\hline 0.60 & 88.33 & 11.18 & 10.00 & 24.15 & 3.05 \\
\hline 0.70 & 89.21 & 8.96 & 11.00 & 24.68 & 4.83 \\
\hline 0.80 & 83.95 & 0.53 & 12.00 & 23.37 & 4.58 \\
\hline 0.90 & 81.28 & 6.70 & 13.00 & 23.31 & 3.45 \\
\hline 1.00 & 72.66 & 0.08 & 14.00 & 25.22 & 5.62 \\
\hline 1.20 & 67.37 & 6.42 & 15.00 & 26.15 & 3.41 \\
\hline 1.65 & 55.07 & 6.92 & 16.00 & 25.17 & 3.28 \\
\hline 2.20 & 44.06 & 0.30 & 17.00 & 22.03 & 4.57 \\
\hline 2.75 & 40.82 & 9.29 & 18.00 & 22.75 & 3.63 \\
\hline 3.50 & 34.88 & 5.67 & 19.00 & 20.83 & 2.41 \\
\hline 4.30 & 33.45 & 3.95 & 20.00 & 20.31 & 1.91 \\
\hline 4.55 & 36.24 & 4.97 & 21.00 & 19.37 & 1.29 \\
\hline 4.80 & 36.52 & 4.61 & 22.00 & 20.30 & 1.08 \\
\hline 5.05 & 34.32 & 4.50 & 23.00 & 19.14 & 1.41 \\
\hline 5.30 & 31.63 & 5.13 & & & \\
\hline
\end{tabular}




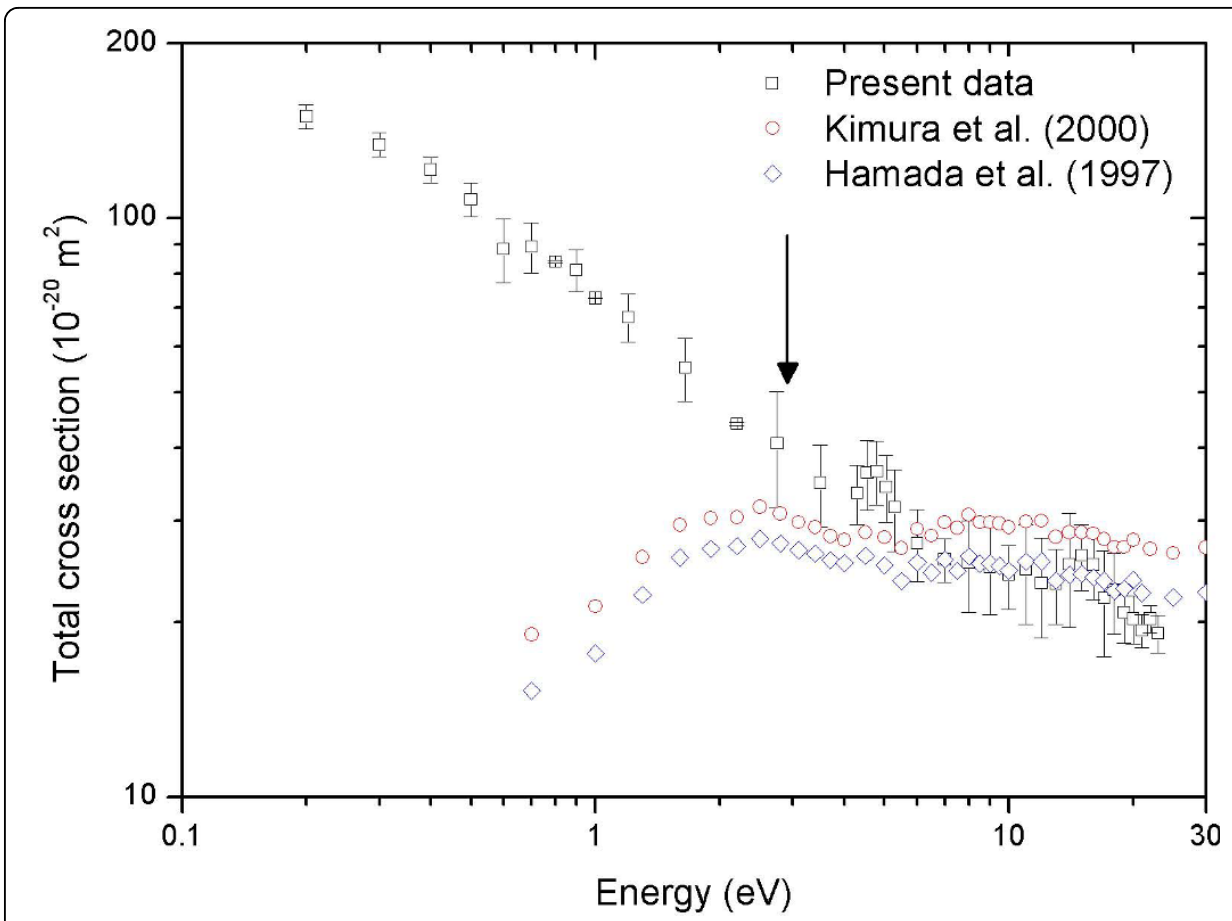

Figure 2 Positron scattering from acetone. Present total cross sections (ㅁ) $\left(\times 10^{-20} \mathrm{~m}^{2}\right)$ for positron scattering from acetone. Also shown are corresponding data from Hamada et al. [11] ( $\diamond)$ and Kimura et al. [12] (0). The arrow on the figure denotes the opening of the Ps formation channel.

Considering figure 2 in more detail we immediately observe that the anticipated low energy behaviour, based on our earlier work [1-5], is indeed found. Namely the magnitude of the TCS increases significantly as the positron impact energy decreases. It is known that the first (direct) ionisation energy of acetone is at $9.71 \mathrm{eV}[19,20]$, implying a positronium formation threshold energy of $2.91 \mathrm{eV}$ for this molecule [21]. Our TCS is seen to monotonically decrease in value with increasing positron energy, until at about $3 \mathrm{eV}$ where there is a change in slope which we believe is consistent with the positronium formation channel opening. A small structure, which was persistently seen during the many crosscheck measurements we made, is also observed at around 4-5 $\mathrm{eV}$. We do not ascribe this small structure to any possible positron binding to the molecule, rather we suggest that it might reflect the opening of one or more of the electronic states of acetone. Thereafter, the TCS again decreases as the energy increases until the opening of the direct ionisation channel.

There are two previous measurements $[11,12]$ against which we can compare the present data, both of which originate from Yamaguchi University. To within the combined uncertainties on the respective data sets, for energies greater than about $6 \mathrm{eV}$, the present TCSs are in good accord with those from Hamada et al. [11] while those from Kimura et al. [12] are a little higher in magnitude. However, as you go to lower positron energies, the discrepancies between the current results and those from the Yamaguchi group [11,12] become progressively worse. For example at $1 \mathrm{eV}$ the present TCS is a factor of $\sim 4$ larger than that of Hamada et al. and a factor of $\sim 3.5$ larger that that of Kimura et al. [12]. We note that a similar effect was recently reported by Szmytkowski [22] in his measurements of total cross sections for electrons scattering 
from acetone, when he compared his data to corresponding results from Yamaguchi University [23]. Makochekanwa et al. [24] recently demonstrated, using theoretical elastic differential cross sections, that for polar molecules the forward angle scattering corrections at low positron energies could be as large as $67 \%$. Assuming the energydependent angular resolution of the Trento apparatus is superior to that of the Yamaguchi spectrometer, which might be reasonable given their apparatus configuration, this effect would explain at least some of the differences between the TCS measurements we see in figure 2. Nonetheless, given the severity of the discrepancies between the Trento and Yamaguchi data below $6 \mathrm{eV}$, we believe the forward angle scattering effect is unlikely to explain all the differences. As a consequence we believe this scattering system would benefit from a further independent experimental study or theoretical investigation (M. H. F. Bettega, 2010, private communication).

\section{Conclusions}

We have reported new total cross section measurements for positron scattering from acetone. The effect of the target molecular dipole moment and dipole polarisability on the scattering dynamics is manifest, particularly at the lower positron impact energies. Indeed when coupled with the recent findings from the San Diego group [6], we see a clear picture emerging for the pivotal role the molecular dipole moment plays in these systems. Agreement with the previous data from Hamada et al. [11] at energies above $6 \mathrm{eV}$ was satisfactory, while significant discrepancies with those data and the results from Kimura et al. [12] at the lower energies were noted. Some of those discrepancies, but not all, we believe are due to the more significant forward angle scattering corrections that need to be applied to the Yamaguchi data compared to the present. We reiterate that a theoretical study of this scattering would be welcome.

\section{Acknowledgements}

This work was supported in part by the Australian Research Council through its Centres of Excellence Programme. We all thank Dr L. Campbell for his help in the preparation of this paper. A.S. also thanks the International Centre for Theoretical Physics - TRIL Program (Italy) for supporting his stay, while M.J.B. thanks the Department of Innovation, Industry, Science and Research for support through their International Science Linkages program. Finally L.C. acknowledges the Provincia Autonoma di Bolzano (Italy) for financial support.

\section{Author details}

${ }^{1}$ Department of Physics, University of Trento, Povo, 1-38123, Trento, Italy. ${ }^{2}$ Department of Physics, Bangabasi Morning College, 19 R. C. Sarani, Kolkata 700009, India. ${ }^{3}$ ARC Centre for Antimatter-Matter Studies, School of Chemical and Physical Sciences, Flinders University, GPO Box 2100, Adelaide, SA 5001, Australia.

Received: 15 July 2010 Accepted: 7 October 2010 Published: 7 October 2010

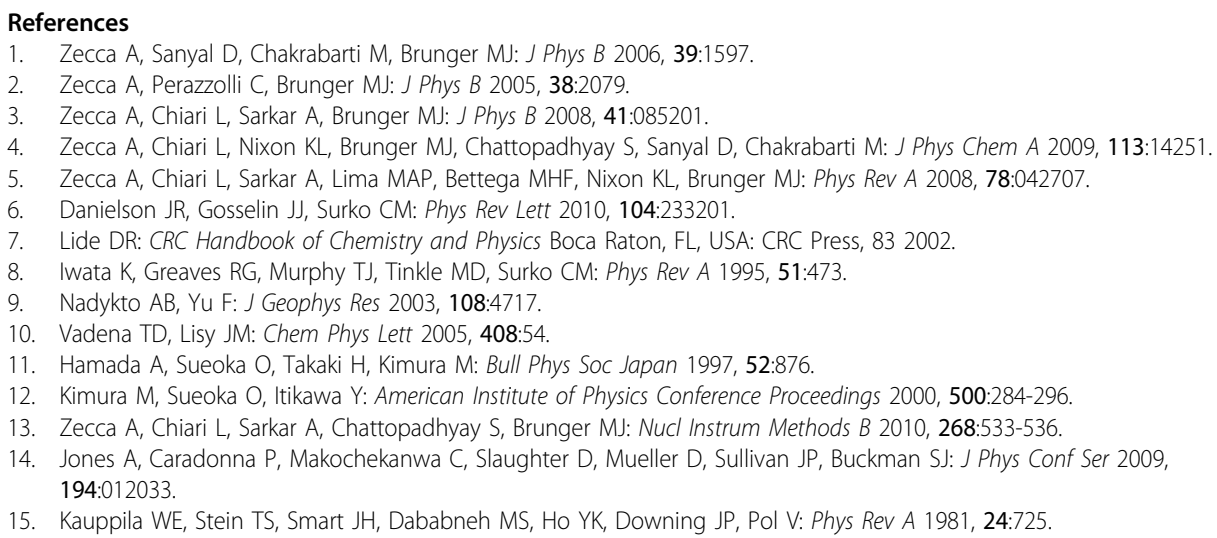


16. Hamada A, Sueoka O: J Phys B 1994, 27:5055.

17. Zecca A, Brunger MJ: In Nanoscale Interactions and Their Applications: Essays in Honour of lan Mc Carthy. Edited by: Wang F, Brunger MJ. Trivandrum, India: Research Signpost; 2007:21-26.

18. Takaishi T, Sensui Y: Trans Faraday Soc 1963, 59:2503-2514.

19. Snyder EM, Buzza SA, Castleman AW Jr: Phys Rev Lett 1996, 77:3347.

20. Traeger JC, McLouglin RG, Nicholson AJC: J Am Chem Soc 1982, 104:5318.

21. Surko C, Gribakin G, Buckman SJ: J Phys B 2005, 38:R57.

22. Szmytkowski C: J Phys B: At Mol Opt Phys 2010, 43:055201.

23. Kimura M, Sueoka O, Hamada A, Itikawa Y: Adv Chem Phys 2000, 111:537.

24. Makochekanwa C, Bankovic A, Tattersall W, Jones A, Caradonna P, Slaughter DS, Nixon K, Brunger MJ, Petrovic Z, Sullivan JP, Buckman SJ: New J Phys 2009, 11:103036.

doi:10.1186/1754-0429-3-4

Cite this article as: Zecca et al.: Total cross section measurements for positron scattering from acetone. PMC Physics B 2010 3:4.

Submit your next manuscript to BioMed Central and take full advantage of:

- Convenient online submission

- Thorough peer review

- No space constraints or color figure charges

- Immediate publication on acceptance

- Inclusion in PubMed, CAS, Scopus and Google Scholar

- Research which is freely available for redistribution 